\title{
Retinal Vascular Changes during the Menstrual Cycle Detected with Optical Coherence Tomography Angiography
}

\author{
Linning Guo, Chenlei Zhu, Ziqi Wang, Zhiqiang Gao, Zongduan Zhang, and Qintuo Pan \\ Eye Hospital and School of Ophthalmology and Optometry, Wenzhou Medical University, Wenzhou, Zhejiang, China \\ Correspondence should be addressed to Qintuo Pan; panqintuo@qq.com
}

Received 22 January 2021; Accepted 2 July 2021; Published 13 July 2021

Academic Editor: Sentaro Kusuhara

Copyright (C) 2021 Linning Guo et al. This is an open access article distributed under the Creative Commons Attribution License, which permits unrestricted use, distribution, and reproduction in any medium, provided the original work is properly cited.

\begin{abstract}
Purpose. To evaluate the effects of the menstrual cycle on the retinal vascular status of healthy women by optical coherence tomography angiography (OCTA). Materials and Methods. Healthy women with regular natural menstrual cycles of 28 to 30 days were recruited for this prospective study. The women's retinal vascular status was measured by OCTA at 3 time points: the early follicular, ovulatory, and midluteal phases of the menstrual cycle. The main outcome measures were foveal avascular zone (FAZ) parameters, perfusion density (PD) percentage in the superficial retinal capillary plexus (SCP), and PD percentage in the deep retinal capillary plexus (DCP). The mean arterial pressure (MAP), spherical equivalent (SE), best-corrected visual acuity (BCVA), intraocular pressure (IOP), and axial (AL) were also measured in a same menstrual cycle. Results. In total, 62 right eyes of 62 women were included in the study. The mean age was $27.0 \pm 1.73$ (range, 24 to 31) years, and the mean menstrual cycle was $28.90 \pm 0.84$ (range, 28 to 30 ) days. The mean values of the DCP-PD parameters were significantly decreased in the nasal and inferior ETDRS subfields during the ovulatory phase. The mean DCP-PD in the nasal ETDRS subfield in the early follicular, ovulatory, and luteal phases was $54.11 \pm 2.85,56.39 \pm 3.03$, and $55.70 \pm 3.27$, respectively. The mean DCP-PD in the inferior ETDRS subfield in the early follicular, ovulatory, and midluteal phases was $52.90 \pm 3.30,54.86 \pm 2.51$, and $55.21 \pm 2.64$, respectively. No significant differences were found in MAP, SE, AL, IOP, FAZ area, or other quadrants of PD parameters, and no significant correlation was found between parameters by OCTA and age, MAP,SE, axial length, or IOP. Conclusions. The DCP-PD decreased in the nasal and inferior ETDRS subfields during the ovulatory phase in our study. This may indicate the need to consider the menstrual phase when interpreting DCP-PD parameters by OCTA in healthy women.
\end{abstract}

\section{Introduction}

Several studies have shown that sex steroid hormone receptors are located in most human ocular tissues, including the cornea, iris, ciliary body, lens, conjunctiva, retina, and lacrimal and meibomian glands [1-5]. Additionally, various endocrine alterations during the menstrual cycle are believed to play a vital role in the vascular physiology and related hemodynamic changes $[6,7]$. Epidemiological studies have shown that sex hormones act differently according to sex and increase the incidence of various ocular diseases including cataract, dry eye, and neovascular agerelated macular degeneration [8-11]. Although some researchers have discovered specific relationships between hormonal fluctuations and ocular tissue variables $[6,11-16]$, other researchers hold opposite opinions [17]. Changes in the corneal thickness, choroidal thickness, intraocular pressure (IOP), and other parameters during the menstrual cycle have been recently reported [12-16]. However, to the best of our knowledge, alterations in the retinal vascular status during the menstrual cycle have not been addressed.

Optical coherence tomography angiography (OCTA) is a recently developed noninvasive imaging technique that provides depth-resolved, high-resolution visualization of the retinal microvasculature and automated or semiautomated quantitative analysis without dye injection. It allows us to easily screen the superficial retinal capillary plexus (SCP) and the deep retinal capillary plexus (DCP) [18-21]. The present study was performed to investigate the retinal vascular changes during the menstrual cycle in healthy reproductive women by OCTA. We considered that if any retinal structures or perfusion parameters are shown to be 
affected by the menstrual cycle, such data should be clinically interpreted with regard to the specific menstrual phase.

\section{Materials and Methods}

This prospective study was conducted from May 1, 2018, through August 31, 2018, at the Wenzhou Medical University Affiliated Eye Hospital. The protocol was approved by the Ethics Board of the Wenzhou Medical University and was performed according to the tenets of the Declaration of Helsinki for research involving human subjects. Signed informed consent forms were returned to the researchers before examinations were performed.

Healthy reproductive women with regular natural menstrual cycles of 28 to 30 days were recruited for this study, and the data from the right eye were selected for analysis. The menstrual cycle history was self-reported by all women and checked for accuracy. The first day (early follicular phase) of menstruation was determined as the day on which bleeding occurred. Because the peak luteinizing hormone (LH) concentration precedes ovulation by 24 to 36 hours, the peak LH concentration in urine was measured when ovulation occurred. All women were supplied with a kit containing five tests (ClearPlan; Unipath Diagnostics, Waltham, MA, USA) that measure the amount of LH in the urine. The test was performed by the participants at home every day until the LH peak occurred. When the test revealed occurrence of the LH peak, the participants were asked to immediately contact the investigators. The next day was determined as the beginning of the ovulatory phase, and 3 to 7 days before the next menstrual bleeding was determined as the beginning of the luteal phase. All the examinations were made at the first day of the early follicular phase, the first day of the ovulatory phase and 3 to 7 days before the next menstrual bleeding.

The inclusion criteria were an age of 15 to 40 years, bestcorrected visual acuity (BCVA) of 20/20 or better, refractive error of $<6$ diopters (D) spherical equivalent (SE), and a regular menstrual cycle. The exclusion criteria were any ocular disease that would prevent examination of the cornea or retina states; a history of laser application or any ocular surgery; a history of smoking or alcohol consumption; any systemic disease such as diabetes mellitus or hypertension; and a history of any medication use within the previous 3 months, including oral contraceptives, hormonal therapy, or systemic vasoactive drugs.

The retinal vascular changes were imaged by using the RTVue XR Avanti OCT device with AngioVue software, version 2016.1.0.26 (Optovue, Inc., Fremont, CA, USA). A detailed ophthalmologic examination, including slit-lamp examination, fundoscopy, BCVA, SE, and intraocular IOP, was performed during each menstrual phase. IOP was measured using Goldmann applanation tonometry, and the axial length (AL) was measured by using a biometer (Lenstar LS 900; Haag-Streit AG, Köniz, Switzerland). All OCTA scans and measurements were performed by an experienced ophthalmic technician three times in the same session with a 10 min interval between each measurement. The intraobserver repeatability of the three measurements was analyzed, and the average value of three repeated measurements was used for the data analysis. A $3 \times 3 \mathrm{~mm}$ cube scan centered on the fovea was acquired containing $304 \times 304$ A-scans. Each OCTA scan was automatically segmented to visualize the retinal perfusion density (PD) of the SCP and DCP. The SCP images were segmented with the inner boundary at the internal limiting membrane and the outer boundary at $10 \mathrm{~mm}$ above the inner plexiform layer (Figure 1(a)). The DCP images were segmented with the inner boundary $10 \mathrm{~mm}$ above the inner plexiform layer and the outer boundary $10 \mathrm{~mm}$ below the outer plexiform layer (Figure 1(b)). For more accurate measurement, the updated optical software (Optovue, Inc.) adopts three-dimensional projection artifact removal algorithms to remove "false" blood flow signals from the DCP. The five regions of interest were based on the Early Treatment Diabetic Retinopathy Study (ETDRS) contours and included the fovea (a circle with a $1 \mathrm{~mm}$ diameter) and the parafovea (an annulus with a $3 \mathrm{~mm}$ outer diameter and a $1 \mathrm{~mm}$ inner diameter centered at the fovea), which were divided into temporal, superior, nasal, and inferior quadrants (Figure 2). The automated software was also used to calculate the area $\left(\mathrm{mm}^{2}\right)$ of the foveal avascular zone (FAZ) (Figure 3). All scans were reviewed to ensure correct segmentation and high image quality (quality index of $>7$ ). Poor-quality scans were excluded. Blood pressure measurements were obtained from the left arm using a sphygmomanometer. The mean arterial pressure (MAP) was obtained by calculating the diastolic pressure plus one-third of the pulse pressure. All examinations were performed within the same menstrual cycle.

\section{Statistical Analysis}

The data were analyzed using the SPSS statistical package, version 21.0 (SPSS Inc., Chicago, IL). For general statistical reporting, the mean values from each dataset were calculated with the standard deviations (SDs). A level of $p<0.05$ was accepted as statistically significant. Visual acuity (VA) data were converted to the $\log$ MAR format for statistical calculations and analyses. The Kolmogorov-Smirnov test was used to check the normality of the sample distribution. The Kaiser-Meyer-Olkin measurement of sampling adequacy and Bartlett's test of sphericity were used to evaluate variance homogeneity. The data were analyzed using the repeated measures analysis of variance with post hoc pairwise comparisons corrected by the Bonferroni method. Greenhouse-Geisser correction and Huynh-Feldt correction were applied to adjust the degrees of freedom. Pearson's coefficient test was used to determine the correlation between parameters. The intraclass correlation coefficient (ICC), values ranging from 0 to 1 , was calculated to determine the repeatability of consecutively measured OCTA. As the ICC value approaches 1 , the repeatability of the measurement increases proportionally.

\section{Results}

Eighty-one volunteers participated in the study and 62 right eyes of 62 healthy women were finally enrolled and the other 


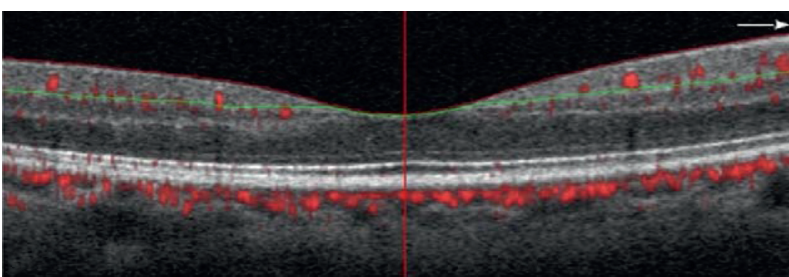

(a)

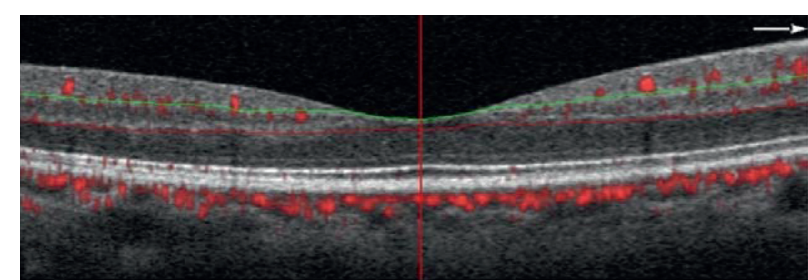

(b)

Figure 1: (a) The superficial retinal capillary plexus between the red and green lines and (b) deep retinal capillary plexus between the green and red lines were detected and separated automatically by using the OCTA instrument.

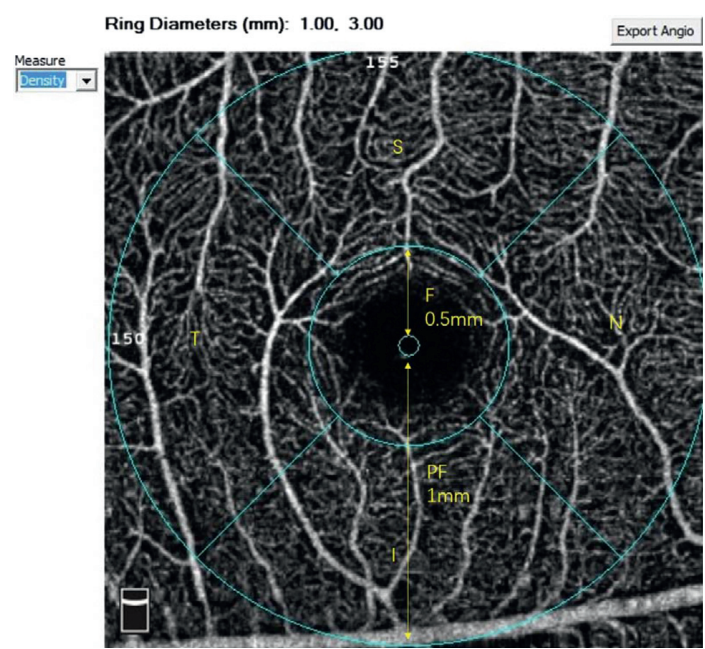

FIgURE 2: Automated superficial capillary plexus PD with overlay of ETDRS subfields. S: superior; I: inferior area; $\mathrm{N}$ : nasal; $\mathrm{T}$ : temporal. F: fovea; and PF: parafovea.

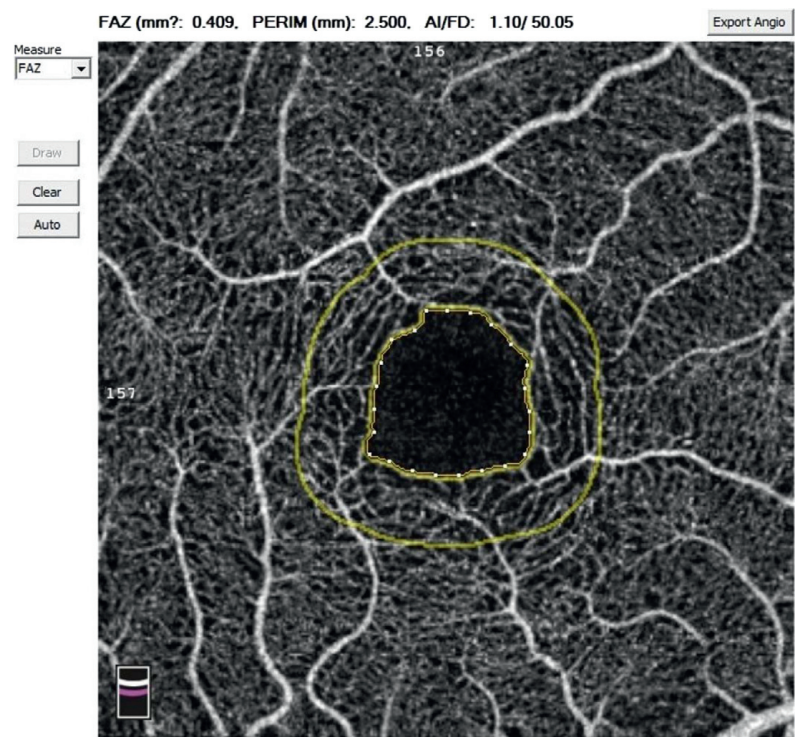

FIGURE 3: Automated FAZ parameters (area, perimeter, acircularity index, and fractal dimension).

19 volunteers did not finish the whole follow-up. None of the women reported any uncomfortable ocular or systemic symptoms. The Kaiser-Meyer-Olkin measure of sampling adequacy was greater than 0.50 (the actual values for each of the studied parameters ranged from 0.665 to 0.870). Bartlett's test of sphericity was $<0.01$ (the actual values for each of the studied parameters were $<0.001)$. The mean age of the participants was $27.0 \pm 1.73$ (range, 24 to 31 ) years with a menstrual cycle of $28.90 \pm 0.84$ (range, 28 to 30) days. The BCVA in all eyes was $20 / 20$ or above and remained constant during the study period. The mean parameters measured values of the 62 women at the 3 time points of the menstrual cycle are reported in Table 1 . There was no significant difference in MAP, SE, AL, IOP, FAZ area, and all quadrants of SCP-PD parameters (Tables 1 and 2). The mean values of the DCP-PD parameters were significantly decreased in the nasal and inferior ETDRS subfields during the ovulatory phase. A significant difference was found in the nasal ETDRS subfield between the early follicular and ovulatory phases and between the ovulatory and luteal phases, but not between the early follicular and luteal phases $(p<0.001$, $p<0.001$, and $p=0.227$, respectively). Similarly, a significant difference was reported in the inferior ETDRS subfield between the early follicular and ovulatory phase and between the ovulatory and luteal phase but not between the early follicular and luteal phase $(p<0.001, p<0.001$, and $p=0.106$, respectively). Detailed data are presented in $\mathrm{Ta}-$ ble 3. No significant correlation between the OCTA parameters and age, MAP, SE, AL, or IOP was found. The statistical analysis demonstrated good intraobserver repeatability of three consecutive measurements in the current study (Table 4).

\section{Discussion}

Various and conflicting findings have been reported about the complex ocular alterations that occur during the menstrual cycle of women, ranging from the anterior segment parameters to choroidal changes, because the eye is also believed to respond to sex hormones. This has raised concern regarding various effects of female hormonal levels on the ocular circulation $[1-5,12-15]$. Studies of cyclic ocular variations in women are scant and discordant. For example, Ulaş et al. [16] found that the choroidal thickness decreased significantly in the midluteal phase by using spectral-domain optical coherence tomography. Because estrogen receptors have been observed mainly in the ganglion cell layer of the retina and occasionally in the choroid [2], we hypothesized that more significant differences would be found in a retinarelated study. However, little information about the retinal 
TABLE 1: Main parameters measured during the menstrual cycle.

\begin{tabular}{lcccc}
\hline Parameter & Mean & SD & SE $^{\mathrm{b}}$ & $p$ \\
\hline SE ${ }^{\mathrm{a}}$ (diopter) & & & & \\
EFP & -3.74 & 1.90 & 0.24 & \\
OVP & -3.75 & 1.91 & 0.24 & 0.44 \\
LP & -3.74 & 1.91 & 0.24 & \\
\hline AL (mm) & & & & \\
EFP & 24.98 & 1.00 & 0.13 & \\
OVP & 24.98 & 0.99 & 0.13 & 0.16 \\
LP & 24.96 & 1.00 & 0.13 & \\
\hline IOP (mmHg) & & & & \\
EFP & 15.16 & 1.74 & 0.22 & \\
OVP & 14.93 & 1.47 & 0.19 & 0.15 \\
LP & 15.38 & 1.19 & 0.15 & \\
\hline LogMAR VA & & & & \\
EFP & 0 & 0 & 0 & \\
OVP & 0 & 0 & 0 & - \\
LP & 0 & 0 & 0 & \\
\hline MAP (mmHg) & & & & \\
EFP & 87.54 & 4.79 & 0.61 & \\
OVP & 87.76 & 4.86 & 0.62 & 0.32 \\
LP & 87.88 & 4.64 & 0.59 & \\
\hline
\end{tabular}

EFP: early follicular phase, OVP: ovulatory phase, LP: luteal phase, $\mathrm{SE}^{\mathrm{a}}$ : spherical equivalent, AL: axial length, IOP: intraocular pressure, VA: visual acuity, MAP: mean arterial pressure, SD: standard deviation, $\mathrm{SE}^{\mathrm{b}}$ : standard error.

vascular changes that occur during the menstrual cycle is available. In this study, we used nonmydriatic, noninvasive OCTA to obtain retinal vascular measurements of women at three points in the menstrual cycle.

Several studies have shown that retinal microvascular changes can be affected by the AL, refractive errors, hypertension, and other factors [21-23]. Mo et al. [21] found that macular flow densities were negatively related to the AL but positively related to BCVA. Von Hanno et al. [22] reported that a higher MAP would reduce the central retinal artery caliber and elevate the central retinal vein caliber. Lee et al. [23] claimed that high blood pressure could affect the retinal microcirculatory structure and function, which may finally affect the retinal thickness. Although, there have been conflicting reports about changes in the blood pressure, associated with changes in the serum osmolality or not, during the menstrual cycle; for example, Chapman et al. [24] reported a decrease in MAP and the serum osmolality for vasodilation during the midluteal phase, while Vokes et al. [25] reported a decrease in the serum osmolality from the follicular phase to the luteal phase without any changes in the blood pressure changes. A hypothesis based on their findings was these changes might be associated with an increase in renal plasma flow and filtration, decreased systemic vascular resistance, or the corpora lutea which could release agents' impact on the hypothalamus to produce a reset osmotic threshold for vasopressin release $[24,25]$. Moreover, Deschenes et al. [26] also reported that estrogen or estrogen and progesterone may help to increase retinal blood flow. Thus, we hypothesized that the AL or SE and the different phases of the menstrual cycle with alterations in hormone levels may induce retinal microvascular changes. In the present study, however, the MAP, BCVA, SE, IOP, AL, and
TABLE 2: FAZ and SCP-PD OCTA parameters measured during the menstrual cycle.

\begin{tabular}{|c|c|c|c|c|}
\hline Parameter & Mean & SD & $\mathrm{SE}^{\mathrm{b}}$ & $p$ \\
\hline \multicolumn{5}{|c|}{ FAZ area $(\mathrm{mm} 2)$} \\
\hline EFP & 0.32 & 0.12 & 0.02 & \multirow{3}{*}{0.34} \\
\hline OVP & 0.33 & 0.12 & 0.01 & \\
\hline LP & 0.32 & 0.12 & 0.01 & \\
\hline \multicolumn{5}{|c|}{ SCP-PD (\%) } \\
\hline \multicolumn{5}{|c|}{ Whole } \\
\hline EFP & 46.30 & 2.81 & 0.36 & \multirow{3}{*}{0.79} \\
\hline OVP & 46.26 & 2.48 & 0.31 & \\
\hline LP & 46.51 & 3.16 & 0.40 & \\
\hline \multicolumn{5}{|l|}{ Parafovea } \\
\hline EFP & 48.94 & 3.83 & 0.49 & \multirow{3}{*}{0.74} \\
\hline OVP & 49.09 & 3.01 & 0.38 & \\
\hline LP & 49.35 & 3.61 & 0.46 & \\
\hline \multicolumn{5}{|l|}{ Fovea } \\
\hline EFP & 16.04 & 5.63 & 0.71 & \multirow{3}{*}{0.30} \\
\hline OVP & 16.53 & 5.60 & 0.71 & \\
\hline LP & 16.58 & 5.59 & 0.71 & \\
\hline \multicolumn{5}{|l|}{ Temporal } \\
\hline EFP & 47.30 & 3.49 & 0.44 & \multirow{3}{*}{0.41} \\
\hline OVP & 46.68 & 4.30 & 0.55 & \\
\hline LP & 47.50 & 3.61 & 0.46 & \\
\hline \multicolumn{5}{|l|}{ Nasal } \\
\hline EFP & 47.79 & 4.59 & 0.58 & \multirow{3}{*}{0.67} \\
\hline OVP & 48.37 & 3.43 & 0.44 & \\
\hline LP & 48.14 & 4.79 & 0.61 & \\
\hline \multicolumn{5}{|l|}{ Superior } \\
\hline EFP & 50.65 & 4.14 & 0.53 & \multirow{3}{*}{0.65} \\
\hline OVP & 50.89 & 2.86 & 0.36 & \\
\hline LP & 51.13 & 3.73 & 0.47 & \\
\hline \multicolumn{5}{|l|}{ Inferior } \\
\hline EFP & 49.98 & 4.65 & 0.59 & \multirow{3}{*}{0.76} \\
\hline OVP & 49.97 & 3.98 & 0.51 & \\
\hline LP & 50.42 & 3.83 & 0.49 & \\
\hline
\end{tabular}

SD: standard deviation, $\mathrm{SE}^{\mathrm{b}}$ : standard error, FAZ: foveal avascular zone, SCP: superficial retinal capillary plexus, PD: perfusion density, EFP: early follicular phase, OVP: ovulatory phase, LP: luteal phase.

SCP-PD did not significantly change during the menstrual cycle, and no significant correlation was found with age, MAP, $\mathrm{SE}, \mathrm{AL}$, or IOP in this homogenous group.

However, we did find that DCP-PD was significantly lower in the nasal and inferior ETDRS subfields during the ovulatory phase than in the early follicular phase and luteal phase. Conversely, we found no significant change in SCP$\mathrm{PD}$. The interrelationships of the retinal capillary plexuses are incompletely understood, and none of the currently available models could fully illustrate the true anatomical structure [27-32]. Some reporters have proposed the morphology of the SCP as a series of "hammocks" between a major artery and vein [28], while the morphology of the DCP appears totally different [29-31]. Garrity et al. [32] proposed that the DCP might be the primary site of venous outflow for the entire retinal microvasculature, while the SCP may not serve as distinct capillary units with an independent arterial supply or independent venous drainage. Thus, we believe the physiologic differences in structure and 
TABLE 3: DCP-PD OCTA parameters measured during the menstrual cycle.

\begin{tabular}{|c|c|c|c|c|}
\hline Parameter & Mean & SD & $\mathrm{SE}^{\mathrm{b}}$ & $p$ \\
\hline \multicolumn{5}{|c|}{ DCP-PD (\%) } \\
\hline \multicolumn{5}{|c|}{ Whole } \\
\hline EFP & 51.67 & 3.52 & 0.45 & \multirow{3}{*}{0.68} \\
\hline OVP & 51.83 & 3.34 & 0.42 & \\
\hline LP & 52.02 & 3.26 & 0.41 & \\
\hline \multicolumn{5}{|l|}{ Parafovea } \\
\hline EFP & 54.70 & 3.54 & 0.45 & \multirow{3}{*}{0.93} \\
\hline OVP & 54.71 & 3.19 & 0.40 & \\
\hline LP & 54.83 & 3.15 & 0.40 & \\
\hline \multicolumn{5}{|l|}{ Fovea } \\
\hline EFP & 31.43 & 8.37 & 1.06 & \multirow{3}{*}{0.70} \\
\hline OVP & 31.74 & 8.50 & 1.08 & \\
\hline LP & 31.69 & 8.14 & 1.03 & \\
\hline \multicolumn{5}{|l|}{ Temporal } \\
\hline EFP & 55.36 & 3.30 & 0.42 & \multirow{3}{*}{0.98} \\
\hline OVP & 55.32 & 3.12 & 0.40 & \\
\hline LP & 55.39 & 3.08 & 0.39 & \\
\hline \multicolumn{5}{|l|}{ Nasal } \\
\hline EFP & 56.39 & 3.03 & 0.38 & \multirow{3}{*}{$<0.001$} \\
\hline OVP & 54.11 & 2.85 & 0.36 & \\
\hline LP & 55.70 & 3.27 & 0.42 & \\
\hline \multicolumn{5}{|l|}{ Superior } \\
\hline EFP & 54.25 & 3.89 & 0.49 & \multirow{3}{*}{0.94} \\
\hline OVP & 54.09 & 3.60 & 0.46 & \\
\hline LP & 54.19 & 3.71 & 0.47 & \\
\hline \multicolumn{5}{|l|}{ Inferior } \\
\hline EFP & 54.86 & 2.51 & 0.32 & \multirow{3}{*}{$<0.001$} \\
\hline OVP & 52.90 & 3.30 & 0.42 & \\
\hline LP & 55.21 & 2.64 & 0.33 & \\
\hline
\end{tabular}

SD: standard deviation, $\mathrm{SE}^{\mathrm{b}}$ : standard error, DCP: deep retinal capillary plexus, PD: perfusion density, EFP: early follicular phase, OVP: ovulatory phase, LP: luteal phase.

TABLE 4: Intraobserver repeatability for the parameters obtained by OCTA.

\begin{tabular}{lc}
\hline Parameter & ICC \\
\hline FAZ area $(\mathrm{mm} 2)$ & \\
EFP & 0.982 \\
OVP & 0.938 \\
LP & 0.911 \\
\hline SCP-PD (\%) & \\
\hline Whole & \\
EFP & 0.954 \\
OVP & 0.917 \\
LP & 0.901 \\
\hline Parafovea & \\
EFP & 0.915 \\
OVP & 0.935 \\
LP & 0.917 \\
\hline Fovea & \\
EFP & 0.971 \\
OVP & 0.877 \\
LP & 0.892 \\
\hline
\end{tabular}

TABle 4: Continued.

\begin{tabular}{|c|c|}
\hline Parameter & ICC \\
\hline \multicolumn{2}{|l|}{ Temporal } \\
\hline EFP & 0.921 \\
\hline OVP & 0.907 \\
\hline LP & 0.884 \\
\hline \multicolumn{2}{|l|}{ Nasal } \\
\hline EFP & 0.925 \\
\hline OVP & 0.947 \\
\hline LP & 0.931 \\
\hline \multicolumn{2}{|l|}{ Superior } \\
\hline EFP & 0.882 \\
\hline OVP & 0.903 \\
\hline LP & 0.885 \\
\hline \multicolumn{2}{|l|}{ Inferior } \\
\hline EFP & 0.896 \\
\hline OVP & 0.904 \\
\hline LP & 0.923 \\
\hline \multicolumn{2}{|c|}{ DCP-PD (\%) } \\
\hline \multicolumn{2}{|c|}{ Whole } \\
\hline EFP & 0.887 \\
\hline OVP & 0.913 \\
\hline LP & 0.873 \\
\hline \multicolumn{2}{|l|}{ Parafovea } \\
\hline EFP & 0.825 \\
\hline OVP & 0.900 \\
\hline LP & 0.910 \\
\hline \multicolumn{2}{|l|}{ Fovea } \\
\hline EFP & 0.883 \\
\hline OVP & 0.902 \\
\hline LP & 0.885 \\
\hline \multicolumn{2}{|l|}{ Temporal } \\
\hline EFP & 0.934 \\
\hline OVP & 0.907 \\
\hline LP & 0.920 \\
\hline \multicolumn{2}{|l|}{ Nasal } \\
\hline EFP & 0.881 \\
\hline OVP & 0.905 \\
\hline LP & 0.917 \\
\hline \multicolumn{2}{|l|}{ Superior } \\
\hline EFP & 0.863 \\
\hline OVP & 0.915 \\
\hline LP & 0.924 \\
\hline \multicolumn{2}{|l|}{ Inferior } \\
\hline EFP & 0.927 \\
\hline OVP & 0.921 \\
\hline LP & 0.892 \\
\hline
\end{tabular}

ICC, intraclass correlation coefficient; SCP: superficial retinal capillary plexus, PD: perfusion density, EFP: early follicular phase, OVP: ovulatory phase, LP: luteal phase, DCP: deep retinal capillary plexus.

function between the SCP and DCP might cause them to be affected differently in the menstrual cycle. We only found differences in DCP-PD in the inferior and nasal subfields, not in the other quadrants, during the ovulatory phase. Chanwimol et al. [33] detected retinal vasculature changes in the third trimester of pregnancy by OCTA, and they found 
that DCP-PD was greater, especially, in the parafoveal, temporal, and inferior subfields. They speculated that this finding may represent gravity-dependent changes. But, whether this phenomenon can explain our results remains unclear because not only do hormones fluctuate in pregnancy but also much greater hemodynamic changes occur in response to the increasing circulatory demand to support the developing fetus over the course of about 9 months [33-36]; this situation seems totally different from that of our study participants. So, this issue remains to be studied in the future.

As mentioned earlier, Munaut et al. [2] reported that estrogen receptors are mainly located in the ganglion cell layer of the retina. Thus, we speculated that the hormone levels during different menstrual phases can explain the significant changes in DCP-PD that occur during the ovulatory phase with the absence of substantial differences in the early follicular and luteal phases. Studies have shown that estradiol is a potent vasodilator, and progesterone is assumed to have the opposite effect $[36,37]$. Deschenes et al. [26] reported that estrogen or estrogen and progesterone together can increase retinal blood flow. Kızıltunç et al. [38] found a significant difference in PD of the whole macula of SCP and DCP in the pregnancy group when evaluating the macular and optic disc vessel density changes in pregnancy by OCTA. Also, the researchers proposed that hormonal changes might be one possible reason; for example, the increased levels of estrogen would lead to vasodilation and decreased vascular resistance, which may result in changes in ocular blood flow. As the estrogen concentration peaks before ovulation and the plasma progesterone concentration usually rise higher during the subsequent luteal phase $[39,40]$, some reporters voiced that the high estrogen levels increased the renin during the subsequent luteal phase $[41,42]$. Thus, we proposed that the increased renin level would induce changes in vascular resistance to influence the blood pressure or ocular flow, which may be detected by OCTA. Measuring the concentrations of serum thyroidstimulating hormone, luteinizing hormone, follicle-stimulating hormone, and prolactin may help to explore and confirm our speculation. However, because this was a noninvasive study, we could not obtain blood samples to search for relationships between hormone concentrations and retinal microvascular changes.

This study had some limitations. Our sample size was limited, and we did not measure the blood hormone concentrations and we could not analysis the peripapillary circulation or the retinal thickness. Further studies with larger sample sizes may help to determine the connections between ocular parameters and serum sex hormone concentrations. Further studies are also needed to investigate the effects of hormone concentrations, other vasoactive agents, and menstrual cycle irregularities. Despite these limitations, our study makes sense in the clinical context given the known menstrual cycle changes that occur in the retinal microvasculature.

In conclusion, this study demonstrated an apparent decreased DCP-PD in the nasal and inferior ETDRS subfields during the ovulatory phase. As the menstrual cycle spans a relatively long period of time in women and the hormones cyclically fluctuate, we tend to consider these findings as normal physiologic fluctuations without adverse effects on ocular function. Therefore, the menstrual phase must be taken into consideration if any significant changes are detected in the partial subfields of DCP by OCTA in women, especially in the nasal and inferior ETDRS subfields.

\section{Data Availability}

The data used to support the findings of this study are available from the corresponding author upon request.

\section{Conflicts of Interest}

The authors declare that they have no conflicts of interest.

\section{Acknowledgments}

This study was supported by research grants from the Wenzhou Public Welfare Social Development (Medical and Health) Science and Technology Projects of China (No. Y20170790).

\section{References}

[1] L. A. Wickham, J. Gao, I. Toda, E. M. Rocha, M. Ono, and D. A. Sullivan, "Identification of androgen, estrogen and progesterone receptor mRNAs in the eye," Acta Ophthalmologica Scandinavica, vol. 78, no. 2, pp. 146-153, 2000.

[2] C. Munaut, V. Lambert, A. Noel et al., "Presence of oestrogen receptor type beta in human retina," British Journal of Ophthalmology, vol. 85, no. 7, pp. 877-882, 2001.

[3] R. J. Handa and R. F. McGivern, "Steroid hormones, receptors, and perceptual and cognitive sex differences in the visual system," Current Eye Research, vol. 40, no. 2, pp. 110-127, 2015.

[4] M. Tachibana, T. Kasukabe, Y. Kobayashi, T. Suzuki, S. Kinoshita, and Y. Matsushima, "Expression of estrogen receptor alpha and beta in the mouse cornea," Investigative Ophthalmology \& Visual Science, vol. 41, no. 3, pp. 668-670, 2000.

[5] P. D. Gupta, K. Johar Sr., K. Nagpal, and A. R. Vasavada, "Sex hormone receptors in the human eye," Survey of Ophthalmology, vol. 50, no. 3, pp. 274-284, 2005.

[6] P. J. Mills, R. A. Nelesen, M. G. Ziegler et al., "Menstrual cycle effects on catecholamine and cardiovascular responses to acute stress in black but not white normotensive women," Hypertension, vol. 27, no. 4, pp. 962-967, 1996.

[7] M. Centofanti, D. Zarfati, G. L. Manni et al., "The influence of oestrogen on the pulsatile ocular blood flow," Acta Ophthalmologica Scandinavica, vol. 78, no. S232, pp. 38-39, 2000.

[8] P. Versura, G. Giannaccare, and E. C. Campos, "Sex-steroid imbalance in females and dry eye," Current Eye Research, vol. 40, no. 2, pp. 162-175, 2015.

[9] P. Versura, M. Fresina, and E. C. Campos, "Ocular surface changes over the menstrual cycle in women with and without dry eye," Gynecological Endocrinology, vol. 23, no. 7, pp. 385-390, 2007.

[10] R. M. Bigsby, S. Valluri, J. Lopez et al., "Ovarian hormone modulation of radiation-induced cataractogenesis: dose- 
response studies," Investigative Opthalmology \& Visual Science, vol. 50, no. 7, pp. 3304-3310, 2009.

[11] J. R. Vingerling, I. Dielemans, J. C. M. Witteman, A. Hofman, D. E. Grobbee, and P. T. V. M. de Jong, "Macular degeneration and early menopause: a case-control study," BMJ, vol. 310, no. 6994, pp. 1570-1571, 1995.

[12] G. Giuffrè, L. D. Rosa, F. Fiorino, D. M. Bubella, and G. Lodato, "Variations in central corneal thickness during the menstrual cycle in women," Cornea, vol. 26, no. 2, pp. 144-146, 2007.

[13] H. Hashemi, S. Mehravaran, and F. Rezvan, "Changes in corneal thickness, curvature, and anterior chamber depth during the menstrual cycle," Canadian Journal of Ophthalmology, vol. 45, no. 1, pp. 67-70, 2010.

[14] G. Seymenoğlu, E. F. Baser, N. Zerdeci, and C. Gülhan, "Corneal biomechanical properties during the menstrual cycle," Current Eye Research, vol. 36, no. 5, pp. 399-403, 2011.

[15] Y. Goldich, Y. Barkana, E. Pras et al., "Variations in corneal biomechanical parameters and central corneal thickness during the menstrual cycle," Journal of Cataract and Refractive Surgery, vol. 37, no. 8, pp. 1507-1511, 2011.

[16] F. Ulaş, U. Doğan, B. Duran, A. Keleş, S. Ağca, and S. Celebi, "Choroidal thickness changes during the menstrual cycle," Current Eye Research, vol. 38, no. 11, pp. 1172-1181, 2013.

[17] P. S. Soni, "Effects of oral contraceptive steroids on the thickness of human cornea," Optometry and Vision Science, vol. 57, no. 11, pp. 825-834, 1980.

[18] S. T. Garrity, N. A. Iafe, N. Phasukkijwatana, X. Chen, and D. Sarraf, "Quantitative analysis of three distinct retinal capillary plexuses in healthy eyes using optical coherence tomography angiography," Investigative Opthalmology \& Visual Science, vol. 58, no. 12, pp. 5548-5555, 2017.

[19] N. Anegondi, L. Chidambara, D. Bhanushali, S. G. K. Gadde, N. K. Yadav, and A. Sinha Roy, "An automated framework to quantify areas of regional ischemia in retinal vascular diseases with OCT angiography," Journal of Biophotonics, vol. 11, no. 2, Article ID e201600312, 2018.

[20] A. La Mantia, R. A. Kurt, S. Mejor et al., "Comparing fundus fluorescein angiography and swept-source optical coherence tomography angiography in the evaluation of diabetic macular perfusion," Retina, vol. 39, no. 5, pp. 926-937, 2019.

[21] J. Mo, A. Duan, S. Chan, X. Wang, and W. Wei, "Vascular flow density in pathological myopia: an optical coherence tomography angiography study," BMJ Open, vol. 7, no. 2, Article ID e013571, 2017.

[22] T. Von Hanno, G. Bertelsen, A. K. Sjølie, and E. B. Mathiesen, "Retinal vascular calibres are significantly associated with cardiovascular risk factors: the Tromsø Eye Study," Acta Ophthalmologica, vol. 92, no. 1, pp. 40-46, 2014.

[23] H.-M. Lee, W.-H. Lee, K. N. Kim, Y. J. Jo, and J. Y. Kim, "Changes in thickness of central macula and retinal nerve fibre layer in severe hypertensive retinopathy: a 1-year longitudinal study," Acta Ophthalmologica, vol. 96, no. 3, pp. e386-e392, 2018.

[24] A. B. Chapman, S. Zamudio, W. Woodmansee et al., "Systemic and renal hemodynamic changes in the luteal phase of the menstrual cycle mimic early pregnancy," American Journal of Physiology-Renal Physiology, vol. 273, no. 5, pp. F777-F782, 1997.

[25] T. J. Vokes, N. M. Weiss, J. Schreiber, M. B. Gaskill, and G. L. Robertson, "Osmoregulation of thirst and vasopressin during normal menstrual cycle," American Journal of Physiology-Regulatory, Integrative and Comparative Physiology, vol. 254, no. 4, pp. R641-R647, 1988.
[26] M. C. Deschênes, D. Descovich, M. Moreau et al., "Postmenopausal hormone therapy increases retinal blood flow and protects the retinal nerve fiber layer," Investigative Opthalmology \& Visual Science, vol. 51, no. 5, pp. 2587-2600, 2010.

[27] M. Paques, R. Tadayoni, R. Sercombe et al., "Structural and hemodynamic analysis of the mouse retinal microcirculation," Investigative Opthalmology \& Visual Science, vol. 44, no. 11, pp. 4960-4967, 2003.

[28] G. Chan, C. Balaratnasingam, P. K. Yu et al., "Quantitative morphometry of perifoveal capillary networks in the human retina," Investigative Opthalmology \& Visual Science, vol. 53, no. 9, pp. 5502-5514, 2012.

[29] I. Gorczynska, J. V. Migacz, R. J. Zawadzki, A. G. Capps, and J. S. Werner, "Comparison of amplitude-decorrelation, speckle-variance and phase-variance OCT angiography methods for imaging the human retina and choroid," Biomedical Optics Express, vol. 7, no. 3, pp. 911-942, 2016.

[30] J. P. Campbell, M. Zhang, T. S. Hwang et al., "Detailed vascular anatomy of the human retina by projection-resolved optical coherence tomography angiography," Scientific Reports, vol. 7, no. 1, p. 42201, 2017.

[31] S. Bonnin, V. Mané, A. Couturier et al., "New insight into the macular deep vascular plexus imaged BY optical coherence tomography angiography," Retina, vol. 35 , no. 11, pp. 2347-2352, 2015.

[32] S. T. Garrity, M. Paques, A. Gaudric, K. B. Freund, and D. Sarraf, "Considerations in the understanding of venous outflow in the retinal capillary plexus," Retina, vol. 37, no. 10, pp. 1809-1812, 2017.

[33] K. Chanwimol, S. Balasubramanian, M. Nassisi et al., "Retinal vascular changes during pregnancy detected with optical coherence tomography angiography," Investigative Opthalmology \& Visual Science, vol. 60, no. 7, pp. 2726-2632, 2019.

[34] P. Soma-Pillay, C. Nelson-Piercy, H. Tolppanen, and A. Mebazaa, "Physiological changes in pregnancy," Cardiovascular Journal of Africa, vol. 27, no. 2, pp. 89-94, 2016.

[35] G. Lockitch and P. R. Gamer, "Clinical biochemistry of pregnancy," Critical Reviews in Clinical Laboratory Sciences, vol. 34, no. 1, pp. 67-139, 1997.

[36] J. G. Ouzounian and U. Elkayam, "Physiologic changes during normal pregnancy and delivery," Cardiology Clinics, vol. 30, no. 3, pp. 317-329, 2012.

[37] J. L. Kirkland, L. Murthy, and G. M. Stancel, "Progesterone inhibits the estrogen-induced expression of c-fos messenger ribonucleic acid in the uterus," Endocrinology, vol. 130, no. 6, pp. 3223-3230, 1992.

[38] P. B. Kizıltunç, B. Varlı, T. Ç Büyüktepe, and H. Atilla, "Ocular vascular changes during pregnancy: an optical coherence tomography angiography study," Graefe's Archive for Clinical and Experimental Ophthalmology=Albrecht von Graefes Archiv für klinische und Experimentelle Ophthalmologie, vol. 258, no. 2, pp. 395-401, 2020.

[39] M. Chidambaram, J. A. Duncan, V. S. Lai et al., "Variation in the renin angiotensin system throughout the normal menstrual cycle," Journal of the American Society of Nephrology, vol. 13, pp. 446-452, 2002.

[40] J. E. Sealey, S. A. Atlas, N. Glorioso, H. Manapat, and J. H. Laragh, "Cyclical secretion of prorenin during the menstrual cycle: synchronization with luteinizing hormone and progesterone," Proceedings of the National Academy of Sciences, vol. 82, no. 24, pp. 8705-8709, 1985.

[41] M. T. Epstein, J. M. Hockaday, and T. D. R. Hockaday, "Migraine and reproductive hormones throughout the 
menstrual cycle," The Lancet, vol. 305, no. 7906, pp. 543-548, 1975.

[42] S. Phillips and B. B. Sherwin, "Variations in memory function and sex steroid hormones across the menstrual cycle," Psychoneuroendocrinology, vol. 17, no. 5, pp. 497-506, 1992. 